\title{
Successful Medical Management of Lymphoma in Six Dogs
}

\author{
M. Bharath, D. Sumathi, G.R. Baranidharan, K. Vijayarani, P. Selvaraj, N. Pazhanivel
}

10.18805/IJAR.B-4457

\begin{abstract}
Background: Lymphoma is considered to be the most common hematopoietic tumor in dogs (83\%) having an overall cure rate of less than five percent. Although many chemotherapy protocols have been suggested, doxorubicin based combination protocols are widely associated with long disease free interval and improved duration of survival. Six dogs presented to Madras Veterinary College Teaching Hospital with clinical signs suggestive of lymphoma were taken up for this study.

Methods: The dogs were subjected to detailed clinical examination and dermatological examination. Fine needle aspiration cytology (FNAC) samples were taken from the lymph nodes in cases of multicentric lymphoma and from cutaneous nodules in cases of cutaneous lymphoma. Tru-cut biopsy and impression smears were collected from the cutaneous nodules. The FNAC and impression smears revealed pleomorphic lymphocytes suggestive of lymphoma. Ultrasonographic examination of all dog revealed the enlargement of mesenteric lymph nodes and spotted appearance of spleen. Treatment with multi-agent chemotherapeutic protocol (University of Wisconsin - Madison, Lymphoma CHOP - 19 protocol) was initiated after confirmative diagnosis.

Result: Remissions in clinical signs were noticed within a week of therapy and no evidence of peripheral lymph node enlargement could be appreciated after three weeks of therapy. The diagnostic and management plans were presented and discussed.
\end{abstract}

Key words: Cytology, Lymphoma, Ultrasound, Wisconsin - Madison protocol.

\section{INTRODUCTION}

Lymphoma is the most frequently diagnosed tumor of dogs which is comparable to the non-Hodgkin's lymphoma in humans (Vail and MacEwen, 2000). A deranged immune system may play a role in lymphomagenesis and few data suggest that autoimmune disorders like immune mediated thrombocytopenia co-occurred with lymphoma in more instances (Keller,1992). Canine lymphoma is one of the most managed tumors of dogs. It is a highly chemo-sensitive tumour having a $65-90 \%$ complete remission rates with a median survival time of 26-51 weeks. Despite multiagent chemotherapy protocols providing complete remission, mortality rates remain higher and it indicated the challenges in their clinical management. The most commonly encountered form of canine lymphoma is the multicentric form, while other forms such as gastro intestinal, mediastinal, cutaneous, ocular, pulmonary and central nervous system lymphomas are also reported (Zandvliet, 2016). Since, usually the dogs are presented in advanced stage of the disease (stage III-IV) require appropriate therapy at the earliest should be initiated to prevent the rapid deterioration of the clinical condition of the animal.

\section{MATERIALS AND METHODS}

Eight dogs presented to the Small Animal Outpatient Medical and Dermatology Unit of the Madras Veterinary College Teaching Hospital during the year 2019-2021 with clinical signs suggestive of lymphoma were taken up for the study. The presenting complaints were anorexia and dullness with swelling in the mandibular region for four dogs and the other two dogs had recurrent non healing wounds without pruritus and occasional bleeding.
Department of Veterinary Clinical Medicine, Madras Veterinary College, Tamil Nadu Veterinary and Animal Sciences University, Chennai-600 007, Tamil Nadu, India.

Corresponding Author: D. Sumathi, Department of Veterinary Clinical Medicine, Madras Veterinary College, Tamil Nadu Veterinary and Animal Sciences University, Chennai-600 007, Tamil Nadu, India. Email: dev_sumi@yahoo.com

How to cite this article: Bharath, M., Sumathi, D., Baranidharan, G.R., Vijayarani, K., Selvaraj, P., Pazhanivel, N. (2021). Successful Medical Management of Lymphoma in Six Dogs. Indian Journal of Animal Research. DOI: 10.18805/IJAR.B-4457.

Submitted: 19-03-2021 Accepted: 11-09-2021 Online: 14-02-2022

Upon clinical examination and dermatological examinations as per standard protocols of Selvaraj et al. (2016) all the eight dogs had enlargement of all the lymph nodes including the inguinal lymph nodes. Fine needle aspiration cytology of the peripheral lymph nodes revealed pleomorphic lymphocytes. Abdominal ultrasound examination was done as per (Sumathi et al., 2017) and it revealed the enlargement of mesenteric lymph node along with the honeycomb pattern in spleen suggestive of splenic involvement and were characterized as grade IV lymphoma subtype 'a'.

Two dogs with non-healing wounds were screened for mites and yeast by skin scrapping and tape impression were found to be negative. Impression cytology taken from the lesions revealed pleomorphic lymphocytes along with mitotic figures suggestive of lymphoma. Ultrasonographic evaluation did not reveal any internal organ involvement. Based on cytology it was confirmed as cutaneous lymphoma. 
For further confirmation punch biopsy samples were submitted for immune-histochemistry.

Complete blood count and serum biochemical parameters were within normal reference range.

\section{Biopsy and histopathological and immuno-histochemical assessment}

A punch biopsy of skin over a cutaneous nodule was taken using $2 \%$ lidocaine infiltration. A punch biopsy tool of $6 \mathrm{~mm}$ diameter was used and the biopsy was taken. Similarly, the nodular lesion was also biopsied. The biopsy materials were fixed in 10\% Formalin. Using standard microtome techniques, histopathological sections were made. Histopathological examination revealed lymphocyte infiltration in epidermis, dermis and around the hair follicles (Fig 1-2). Immuno-histochemical assessment was done by using CD3+ and CD79a markers using the protocols of day (1995). CD3+ was found to be highly expressed (Fig 3-4). On the basis of these clinical and laboratory testing findings, the dogs were diagnosed to have Epitheliotrophic $T$ cell lymphoma derived from $\mathrm{CD} 3+$ positive cells.

\section{Clinical management and outcome}

The prognosis was informed to the owner and imparting knowledge about the treatment, its side effects and the possible outcomes were done before initiation of therapy. All the dogs were treated as per Madison-Wisconsin protocol based on Cyclophosphamide, Hydroxydaunorubicin (doxorubicin) along with Oncovin (vincristine) and

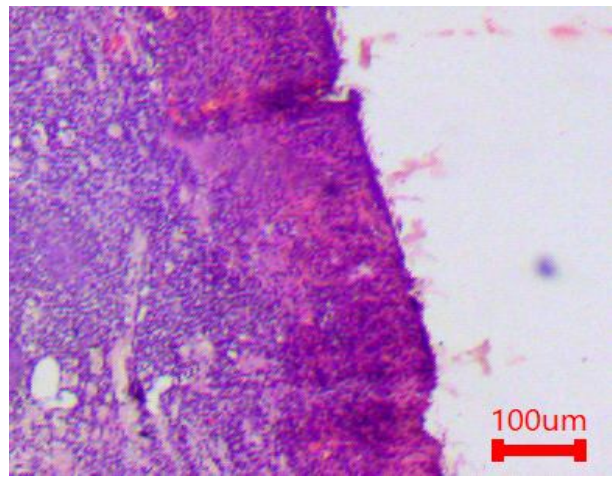

Fig 1 and 2: Histopathological feature of a lesion - Neoplastic lymphocytes in dermis and epidermis (H\&E-4x) and (H\&E-10x).

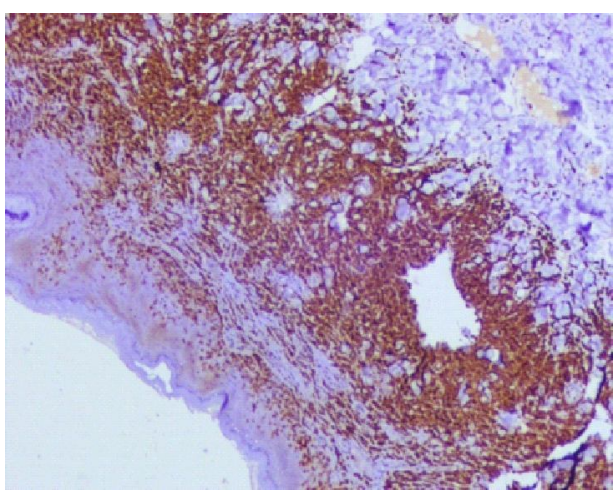

$\mathrm{IHC}-4$

Fig 3 and 4: The neoplastic cells are immuno-labelled for anti CD3 marker with brown cytoplasmic stain.

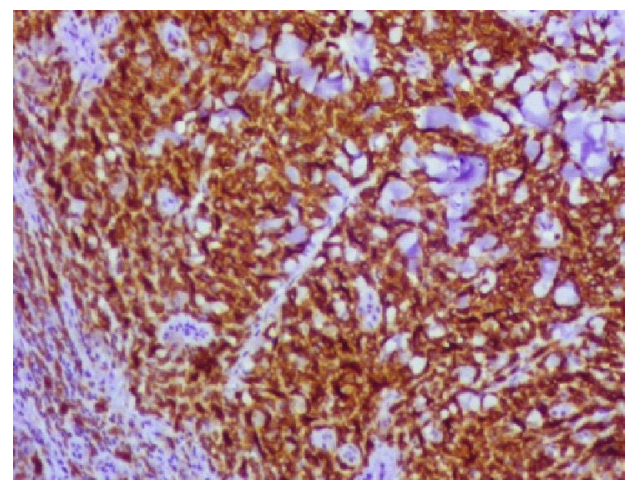

$\mathrm{IHC}-10 \mathrm{X}$

prednisolone (CHOP). Treatment was initiated with vincristine at the dose rate of $0.5 \mathrm{mg} / \mathrm{m}^{2}\left(0.4-0.6 \mathrm{mg} / \mathrm{m}^{2}\right)$ along with prednisolone at $2 \mathrm{mg} / \mathrm{kg}$ PO SID for one week. Prednisolone was tapered weekly over a period of four weeks while vincristine was administered in alternate weeks. Cyclophosphamide at $250 \mathrm{mg} / \mathrm{m}^{2}$ was administered at the second week and once in four weeks while Doxorubicin at $30 \mathrm{mg} / \mathrm{m}^{2}$ was administered at the fourth week and once in four weeks. The protocol was continued for 19 weeks.

Haematology and biochemistry were taken one day prior to the scheduled dosing. One dog had thrombocytopenia $(50,000 / \mu l)$ during the third week and hence chemotherapy was postponed till the thrombocytes resolved to normal level. After a week the platelet had improved to $1,40,000 / \mu \mathrm{l}$ and the therapy was resumed. The WBC count was stable during pre and post treatment period. The pre and post chemotherapy haematology and biochemistry values were presented in Table 1 and 2 respectively. No significant changes in the mean values were reported except for creatinine which showed significant increase post chemotherapy, but the values were within the normal limit.

Owing to the old age of two dogs with cutaneous lymphoma and its extensive metastasis, one dog collapsed within three days of initiation of therapy and one dog had excellent resolution of lesions till the third week, had sudden flare up of nodules and collapsed before it could reach the fourth week.

All the other six dogs survived till the $19^{\text {th }}$ week of treatment. Clinical remission as described as complete

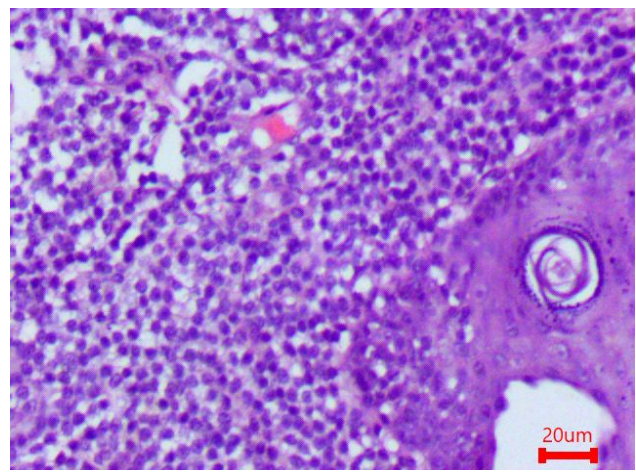


Successful Medical Management of Lymphoma in Six Dogs

Table 1: Haematology Parameters.

\begin{tabular}{lccc}
\hline & Pre-treatment & Post treatment & T value \\
\hline $\mathrm{Hb}(\mathrm{g} / \mathrm{dl})$ & $10.5 \pm 1.09$ & $9.35 \pm 0.33$ & $1.168^{\mathrm{NS}}$ \\
$\mathrm{PCV}(\%)$ & $30.45 \pm 3.03$ & $27.5 \pm 2.09$ & $0.989^{\mathrm{NS}}$ \\
$\mathrm{RBC}\left(\times 10^{6} \mathrm{cell} / \mathrm{sl}\right)$ & $4.94 \pm 0.52$ & $4.40 \pm 0.26$ & $1.217^{\mathrm{NS}}$ \\
$\mathrm{WBC}(\mathrm{cell} / \mathrm{\mu l})$ & $15400 \pm 3831$ & $15025 \pm 3922$ & $0.069^{\mathrm{NS}}$ \\
Platelets $(/ \mu \mathrm{l})$ & $480250 \pm 68532$ & $201000 \pm 70904$ & $2.338^{\mathrm{NS}}$ \\
Neutrophils $(\%)$ & $79.5 \pm 4.73$ & $73.25 \pm 2.49$ & $0.905^{\mathrm{NS}}$ \\
Lymphocytes $(\%)$ & $15.75 \pm 4.55$ & $20.75 \pm 2.51$ & $-0.736^{\mathrm{NS}}$ \\
Monocytes $(\%)$ & $5 \pm 0.40$ & $5.25 \pm 0.62$ & $-0.522^{\mathrm{NS}}$ \\
\hline
\end{tabular}

Table 2: Biochemical Parameters.

\begin{tabular}{lccc}
\hline & Pre-treatment & Post treatment & T value \\
\hline Glucose $(\mathrm{mg} / \mathrm{dl})$ & $88 \pm 13.9$ & $107.8 \pm 6.3$ & $-1.54^{\mathrm{NS}}$ \\
BUN $(\mathrm{mg} / \mathrm{dl})$ & $8.2 \pm 1.05$ & $16.6 \pm 3.80$ & $-2.38^{\mathrm{NS}}$ \\
Creatinine $(\mathrm{mg} / \mathrm{dl})$ & $0.6 \pm 0.12$ & $1 \pm 0.47$ & $-3.76^{*}$ \\
Total protein $(\mathrm{g} / \mathrm{dl})$ & $7.1 \pm 0.27$ & $7.2 \pm 0.23$ & $-0.36^{\mathrm{NS}}$ \\
Albumin $(\mathrm{g} / \mathrm{dl})$ & $2.8 \pm 0.08$ & $2.6 \pm 0.24$ & $0.73^{\mathrm{NS}}$ \\
Calcium $(\mathrm{mmol} / \mathrm{dl})$ & $10.5 \pm 0.27$ & $9.3 \pm 0.42$ & $1.97^{\mathrm{NS}}$ \\
Phosphorus $(\mathrm{mg} / \mathrm{dl})$ & $3.9 \pm 0.51$ & $5.2 \pm 0.32$ & $-3.20^{\mathrm{NS}}$ \\
ALP $(\mathrm{IU} / \mathrm{l})$ & $116 \pm 18$ & $303 \pm 52$ & $-2.70^{\mathrm{NS}}$ \\
ALT $(\mathrm{IU} / \mathrm{l})$ & $27.5 \pm 2.3$ & $213 \pm 70.01$ & $-2.69^{\mathrm{NS}}$ \\
\hline
\end{tabular}

absence of clinical signs suggesting lymphoma was achieved in all dogs within the third week of therapy. Lymph nodes were normal on physical examination and no mesenteric lymph nodes are visualized in abdominal ultrasound.

\section{RESULTS AND DISCUSSION}

Among canine malignant tumours lymphoma is considered to be the most frequently diagnosed disease. In spite of this, it is considered to be the most commonly managed tumours in veterinary oncology with the incidence rates of 13-114 dogs per 100,000 dogs annually (Dorn et al., 1967; Teske, 1994; Dobson et al., 2002). Though the treatment option is not curative, it is considered to be palliative and improve the quality of life considerably also prolonging the life span. Though, canine lymphoma is considered as a single disease, many clinically and morphologically distinct forms of the disease occur. The incidence of lymphoma is more in medium sized to larger breeds (Teske et al., 1994; Edwards et al., 2003; Villamil et al., 2009) and affects the middle aged to older age group animals with a incidence of 1.5 case per 100,000 dogs in $<1$ year of age to 84 cases per 100,000 dogs in $>10$ years of age group (Dorn et al., 1967).

The most commonly reported clinical presentation of canine lymphoma is the multicentric form which accounts for about $75 \%$ of all canine lymphoma cases (Ponce et al., 2010; Vezzali et al., 2010). The World Health Organization (WHO) stages for canine multicentric lymphoma are as follows, (Owen 1980).

\section{Stage}

- Stage I: Single node or lymphoid tissue in single organ (excluding bone marrow).

- Stage II: Regional involvement of multiple lymph nodes ( \pm tonsils).

- Stage III: Generalized lymph node involvement.

- Stage IV: Stage I - III with involvement of liver and/or spleen.

- Stage V: Stage I - IV with involvement of blood or bone marrow.

\section{o Substage}

a: Absence of systemic signs.

b: Presence of systemic signs (fever, $>10 \%$ weight loss, hypercalcemia).

Epitheliotropic T-cell lymphoma, especially that involving cutaneous forms in the dog are rare neoplastic conditions. Such cases have unknown aetiology and till date no specific reasons were found. Epitheliotropic lymphoma mainly consists of CD3-positive T-cells, which usually express CD8 (Moore et al., 1994). Canine cutaneous lymphoma is observed to be a relatively rare disease and is histologically classified into epitheliotropic and nonepitheliotropic forms. Cutaneous lymphoma is typically a T-cell lymphoma and more frequently epitheliotropic than non-epitheliotropic (Day, 1995). Cutaneous epitheliotropic T-cell lymphoma typically presents as a chronic multifocal skin disease, as observed in this study; affections on the mucous membranes (especially buccal) and muco-cutaneous junctions are also documented (Moore et al., 2009). Similar lesions were also evident in this study (Fig 1-3).

The skin lesions always have varied presentations. Cutaneous epitheliotropic T-cell lymphoma typically presents as a chronic multifocal skin disease, but can also affect the mucous membranes (especially buccal) and mucocutaneous junctions (Moore et al., 2009) and affected dogs are usually presented with a history of a chronic dermatitis and lesions reported Erythema, Plaques, Scaling, Nodules, Erosion/ulceration, Crusting, Mucosal lesions, Pruritus and Papules as reported by Fontaine et al. (2010). The lesions reported in this case concurred with the reports of the authors.

The characteristic lesion, common to all forms of CETL, is the tropism of neoplastic cells for the epidermis and the adnexal structures (hair follicles, apocrine sweat and sebaceous glands) was reported by Scott (2001) and Gross et al. (2008). In this rare disease, the dermatosis is observed to be characterized by infiltration of neoplastic T lymphocytes with a specific tropism for the epidermis and adnexal structures. These require specialised diagnostic testing. Cytology, biopsy, histopathology and immuno-histochemical studies only helped to confirm the disease, as observed in this study. In human beings, the disease was divided into three sub-forms as Mycosis fungoides (MF), Pagetoid reticulosis (PR) and Sézary syndrome (SS). Similar categorization is also practiced in dogs. The importance lies in the differentiation from non-epitheliotropic lymphoma (NEL) or dermal lymphoma. NEL can be primary (dermal origin) or secondary to a disseminated lymphoma. 
The diagnosis of lymphoma has been largely based on Fine needle aspiration cytology (FNAC) of the peripheral lymph node which is considered to be a quick, accurate and cost effective method to diagnose lymphoma (Marconato, 2011). Use of flow-cytometric immunophenotyping increases the diagnostic potential of FNAC and sub classification of high grade lymphoma thereby avoiding the need for invasive surgical biopsies (Gelain et al., 2008). The most commonly used antibodies for B-cell lymphoma were CD20, CD21, CD79a and PAX5 while CD3, CD4 and CD8 for T-cell lymphomas (Caniatti et al., 1996). Immunophenotyping on histological samples using CD3 and CD79a antibodies are considered to be sufficient for routine patient care. Abdominal and thoracic radiographs can be used to in case if multicentric lymphoma to asses possible metastasis and involvement of mediastinal, mesenteric and inguinal lymph node involvement. $70 \%$ of thoracic radiographs of canine multicentric lymphoma reveal lymphadenopathy, pulmonary infiltration and the presence of cranial mediastinal mass (Starrak et al., 1997). Abdominal ultrasonography of abdomen is helpful in assessing the lymph node size and architecture (Nyman et al., 2005) and also the presence of splenic and hepatic involvement (Crabtree et al., 2010).

Due to the systemic presentation of canine lymphoma, chemotherapy stands to be mainstay of treatment. Though many single agent and multi-agent protocols have been proposed and followed, doxorubicin based CHOP (cyclophosphamide, Hydroxydaunorubicin (doxorubicin), Oncovin (vincristine) and prednisolone) protocol is considered to provide highest response rates and hence used in many protocols used to treat high grade lymphoma (Zandvliet 2016). In this case the treatment CHOP based University of Wisconsin - Madison protocol was followed.

T-cell lymphomas generally have a poor prognosis when treated with CHOP based chemotherapeutic protocol on comparison to B-cell lymphomas (Thamm, 2019). This was evident from our study also in which the cutaneous T-cell lymphoma dogs does not quite respond to therapy and succumbed to disease while the multicentric lymphoma dogs responded well survived through the entire course of disease.

\section{REFERENCES}

Caniatti, M., Roccabianca, P., Scanziani, E., Paltrinieri, S. and Moore, P.F. (1996). Canine Iymphoma: Immunocytochemical analysis of fine-needle aspiration biopsy, Veterinary Pathology. 33(2): 204-212.

Crabtree, A.C., Spangler, E., Beard, D. and Smith, A. (2010). Diagnostic accuracy of gray-scale ultrasonographyfor the detection of hepatic and splenic lymphoma in dogs. Veterinary Radiology and Ultrasound. 51(6): 661-664.

Day, M.J. (1995). Immunophenotypic characterization of cutaneous lymphoid neoplasia in the dog and cat. Journal of Comparative Pathology. 112(1): 79-96.

Dobson, J.M., Samuel, S., Milstein, H., Rogers, K. and Wood, J.L.N. (2002). Canine neoplasia in the UK: Estimates of incidence rates from a population of insured dogs, The Journal of Small Animal Practice. 43(6): 240-246.
Dorn, C.R., Taylor, D.O. and Hibbard, H.H. (1967). Epizootiologic characteristics of canine and feline leukemia and lymphoma., American Journal of Veterinary Research. 28(125): 9931001.

Edwards, D.S., Henley, W.E., Harding, E.F., Dobson, J.M. and Wood, J.L.N. (2003). Breed incidence of lymphoma in a UK population of insured dogs, Veterinary and Comparative Oncology. 1(4): 200-206.

Fontaine, J., Heimann, M. and Day, M.J. (2010). Canine cutaneous epitheliotropic T-cell lymphoma: A review of 30 cases, Veterinary Dermatology. 21(3): 267-275.

Gelain, M.E., Mazzilli, M., Riondato, F., Marconato, L. and Comazzi, S. (2008). Aberrant phenotypes and quantitative antigen expression in different subtypes of canine lymphoma by flow cytometry, Veterinary Immunology and Immunopathology. 121(3-4): 179-188.

Gross, T.L., Ihrke, P.J., Walder, E.J. and Affolter, V.K., (2008). Skin diseases of the dog and cat: Clinical and histopathologic diagnosis. John Wiley and Sons.

Keller, E.T., (1992). Immune-mediated disease as a risk factor for canine lymphoma., Cancer. 70(9): 2334-7.

Marconato, L., (2011). The staging and treatment of multicentric highgrade lymphoma in dogs: A review of recent developments and future prospects, The Veterinary Journal. 188(1): 34-38.

Moore, P.F., Affolter, V.K., Graham, P.S. and Hirt, B. (2009). Canine epitheliotropic cutaneous T-cell lymphoma: An investigation of T-cell receptor immunophenotype, lesion topography and molecular clonality, Veterinary Dermatology. 20(5-6): 569-576.

Moore, P.F., Olivry, T. and Naydan, D. (1994). Canine cutaneous epitheliotropic lymphoma (mycosis fungoides) is a proliferative disorder of CD8+ T cells, The American Journal of Pathology. 144(2): 421-429.

Nyman, H.T., Kristensen, A.T., Skovgaard, I.M. and McEvoy, F.J. (2005). Characterization of normal and abnormal canine superficial lymph nodes using gray-scale B-mode, color flow mapping, power and spectral doppler ultrasonography: A Multivariate Study, Veterinary Radiology and Ultrasound. 46(5): 404-410.

Owen (1980). TNM Classification of Tumours in Domestic Animals. World Health Organisation. pp 1-52.

Ponce, F., Marchal, T., Magnol, J.P., Turinelli, V., Ledieu, D., Bonnefont, C., Pastor, M., Delignette, M.L. and Fournel-Fleury, C. (2010). A morphological study of 608 cases of canine malignant lymphoma in France with a focus on comparative similarities between canine and human lymphoma morphology, Veterinary Pathology. 47(3): 414-433.

Selvaraj, P., Srinivasan, S.R., Nambi, A.P. and Nagarajan, B. (2016). Endocrine Dermatoses of Testicular Tumor Origin: Incidence, Clinical and Clinico-Pathological Status and Outcome. Indian Veterinary Journal. 93: 83-84.

Scott (2001). Immune Mediated Disorders. Muller and Kirk's Small Animal Dermatology. pp 667-779.

Starrak, G.S., Berry, C.R., Page, R.L., Johnson J.L. and Thrall, D.E. (1997). Correlation between thoracic radiographic changes and remission/survival duration in 270 dogs with lymphosarcoma, Veterinary Radiology and Ultrasound. 38(6): 411-418. 
Sumathi, D., Prathaban, S., Selvaraj, P., Dhanapalan, P., Murali Manohar, B. and Kumanan, K. (2017). 2D and 3D ultrasonographic study of hepatobiliary disorders in dogs and their etiological pattern Indian. J. Vet. Med. 37: 1-8.

Teske, E., (1994). Canine malignant lymphoma: a review and comparison with human non-Hodgkin's lymphoma. The Veterinary Quarterly. 209-219.

Teske, E., de Vos, J.P., Egberink, H.F. and Vos, J.H. (1994). Clustering in canine malignant lymphoma. The Veterinary Quarterly. 16(2): 134-136.

Thamm, D.H., (2019). Novel Treatments for Lymphoma, Veterinary Clinics of North America - Small Animal Practice. 49(5): 903-915.
Vail, D.M. and MacEwen, E.G. (2000). Spontaneously occurring tumors of companion animals as models for human cancer, Cancer Investigation. 18(8): 781-792.

Vezzali, E., Parodi, A.L., Marcato, P.S. and Bettini, G. (2010). Histopathologic classification of 171 cases of canine and feline non-Hodgkin lymphoma according to the WHO, Veterinary and Comparative Oncology. 8(1): 38-49.

Villamil, J.A., Henry, C.J., Hahn, A.W., Bryan, J.N., Tyler, J.W. and Caldwell, C.W. (2009). Hormonal and Sex Impact on the Epidemiology of Canine Lymphoma, Journal of Cancer Epidemiology. 1-7.

Zandvliet, M., (2016). Canine lymphoma: A review, The Veterinary Quarterly. 36(2): 76-104. 